\title{
Shifting and Pinning of a Magnetic Vortex Core in a Permalloy Dot by a Magnetic Field
}

\author{
Thomas Uhlig, ${ }^{*}$ M. Rahm, Christian Dietrich, Rainer Höllinger, Martin Heumann, Dieter Weiss, and Josef Zweck ${ }^{\dagger}$ \\ Institut für Angewandte Physik, Universität Regensburg, 93040 Regensburg, Germany
}

(Received 1 February 2005; published 29 November 2005)

\begin{abstract}
Magnetic pinning in thin films seems to be a major research subject in the near future, as it is involved in all switching processes which include a movement of a domain wall or a magnetic vortex. We used Lorentz transmission electron microscopy and vortex pinning at artificial pinning sites to investigate the pinning behavior of magnetic vortices for the first time with high spatial resolution.
\end{abstract}

PACS numbers: 75.60.Jk, 68.37.Lp

Because of Brown's paradoxon [1] low switching fields in magnetic storage devices [2] can be achieved, if the process of changing the stored information does not involve domain wall nucleation or annihilation.

For magnetic storage, circular ring structures have been proposed [3] and investigated extensively. An overview of the related works can be found in [4]. In the following we will use the term " $n$-stage switching process" for the results of the here described experiment: An in-plane external magnetic field, which saturates the particle is applied. Then the field is lowered to zero and increased in the opposite direction until saturation is reached. If during this process a fundamental change of the basic micromagnetic configuration is observed, this is seen as an additional stage of the process.

While for ring structures with a large inner to outer diameter relation a two-stage switching process was found [5], for a small ratio a process involving three stages has been reported [6]. Here, coming from a saturated state, a flux closure state is generated when lowering the external field, which can be seen as a vortex state [7] with the vortex core located in the central hole. At a certain opposite field value the vortex core jumps out of the central hole and is visible in the ring. A third switching process then leads to the final saturated state.

Related behavior is found for dots containing a tiny hole as a pinning site [8]. There the corresponding switching process involves four stages. After the vortex nucleation (1) the vortex is only shifted (no change in the basic configuration), then it disappears in the hole. (2) In the opposite field the vortex renucleates (3), is shifted, and finally converts into a single domain state (4). Including two or more pinning sites in the disk allows bistable or multistable switching [9].

For such devices a detailed knowledge of the vortex core movement in circular magnetic disks is of great importance. One frequently used approach to a theoretical description is the application of the so-called rigid vortex model [10] which describes the movement of a magnetic vortex under an external magnetic field, based on assumptions given in [10]. We emphasize that the model does not explain the nucleation of a vortex. Thus, in our investiga- tions we always use magnetic configurations with a vortex already present.

Although frequently used, an experimental investigation of the range of validity of this model has not been done so far, mostly due to imaging techniques that are too insensitive. In this work we investigated this movement caused by external magnetic fields using differential phase contrast microscopy (DPC) [11], a high-resolution imaging technique for micromagnetic structures, which is a Lorentz transmission electron microscopy method. For the first time, vortex movements and pinning behavior has been observed with high spatial resolution $(5 \mathrm{~nm})$ as defined by the electron probe diameter of our microscope, a FEI Tecnai F30 (Regensburg special). Our measurements are compared with calculations based on the rigid vortex model [10].

The samples are prepared on a 30-nm-thick electron transparent $\mathrm{Si}_{3} \mathrm{~N}_{4}$ membrane. A 20 -nm-thick Permalloy layer is deposited by thermal evaporation, capped by $5 \mathrm{~nm}$ aluminum to prevent oxidation. The Permalloy has been found to be polycrystalline with an average crystallite size of 5-10 nm. Polymethylmethacrylat (PMMA) is used as resist, which is exposed via electron beam lithography employing the nanonic eLitho EBL system. After development, titanium is deposited. The thickness depends on the relative argon ions etching rate of Permalloy and Ti. During the etching the Permalloy must be removed completely in non-Ti covered areas, while the Ti mask must remain. This led, in our case, to a Ti thickness of $11 \mathrm{~nm}$. After a subsequent lift-off procedure the desired pattern remains as $\mathrm{Ti}$ structures on the Permalloy layer. This defines an etching mask for the argon ion etch process, which transfers the pattern to the Permalloy.

For the pinning investigations with artificially introduced holes, an additional patterning process is necessary, because with the conventional preparation method described above it is very difficult to unhinge inner parts of a structure on a membrane in the lift-off process. The second patterning avoids the critical lift-off step.

Here, in completed Permalloy, disks holes were introduced by a second patterning process (Fig. 1). The specimen is once again covered with PMMA, the positions of 
a

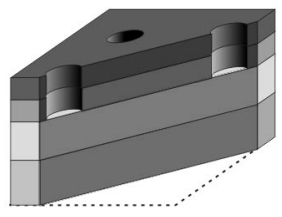

Coating, patterning and developing of PMMA resist onto the prepatterned specimen

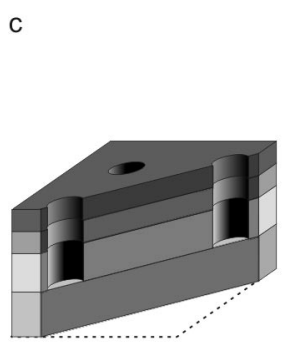

After ion beam etching

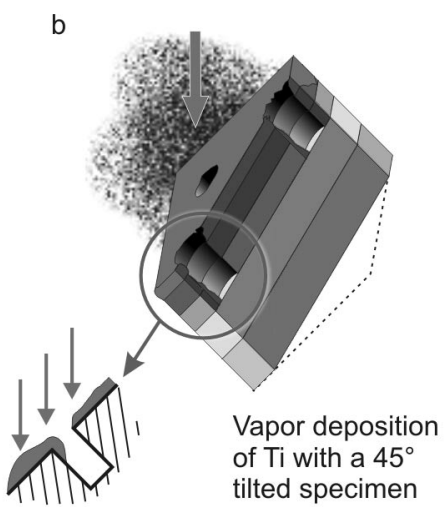

d

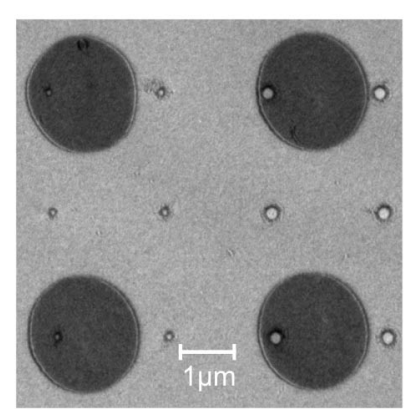

FIG. 1. In a second preparation step a hole array was introduced in the already patterned Permalloy disks. First, the sample was coated with PMMA, the hole pattern was transferred via $e$-beam lithography and the resist developed (a). Then Titanium was deposited onto the specimen, tilted by $45^{\circ}$ (b). This results in an incomplete coating, which leads to a selective etching of the holes during the ion etching process (c). After removal of the residual PMMA and Ti, the hole pattern was transferred to the disks as well as to the electron transparent substrate (d). A suitable alignment of the two preparation steps leads to central holes in the disks.

the holes are transferred to the resist via $e$-beam lithography, followed by development [Fig. 1(a)]. Now titanium is evaporated as an etch mask, while the specimen is oriented in a $45^{\circ}$ angle with respect to the Ti-beam direction. This prevents the titanium from covering the base of the holes and allows the definition of the etch mask without the critical lift-off process [Fig. 1(b)]. After a subsequent argon ion etch process [Fig. 1(c)] the hole pattern is transferred to the circular dots [Fig. 1(d)], the residual Ti and PMMA is removed in acetone.

First, a circular magnetic disk with a radius $r=1.5 \mu \mathrm{m}$ with vortex configuration was investigated under the influence of an external magnetic field $H_{\text {ext }}$. The rigid vortex model [10] allows for small deviations $d \ll r$ from the center of the disk the calculation of the vortex position [12],

$$
\frac{d}{r}=\frac{H_{\mathrm{ext}}}{M_{S}}\left[\frac{h}{2 \pi r}\left(\ln \frac{8 r}{h}-\frac{1}{2}\right)-\frac{l_{\mathrm{ex}}^{2}}{r^{2}}\right]^{-1},
$$

where $M_{s}$ is the saturation magnetization, $h$ the particle

height, and $l_{\mathrm{ex}}$ the exchange length, which is $\approx 5.7 \mathrm{~nm}$ for Permalloy. Equation (1) was derived comparing the involved energy densities. This gives a direct proportionality of the vortex $d$ shift and the applied external field $H_{\text {ext }}$, which can also be observed in the experiment. As will become obvious below, Eq. (1) is also an excellent approximation for even larger values for $d$.

In Fig. 2 the vortex position determination by a DPC line scan is visualized exemplarily by a scan over a dot with a central hole. The scan signal contains both the structural (letters $a, b$ in Fig. 2) and the magnetic signal. The change in the magnetic signal allows a very precise localization of the vortex position (letter $c$ in Fig. 2) with an accuracy of about $50 \mathrm{~nm}$. This accuracy of only $50 \mathrm{~nm}$ compared to the resolution of the imaging technique used $(5 \mathrm{~nm})$ is due to the fact that a simple line scan cannot ensure unambiguously that the vortex core has been probed exactly. From [13] it is known, however, that for this type of material typical vortex diameters lie in the range of $15-20 \mathrm{~nm}$ (FWHM), the value given above corresponds to a rather pessimistic estimation of the actual position.

In Fig. 3 the vortex position is plotted versus the applied field. The graph shows approximately a linear behavior in the central region around zero field, as expected from Eq. (1). Approaching the particle's edges the slope in Fig. 3 decreases, which reveals that the vortex movement is hampered near the boundary. This confirms that the validity of the rigid vortex model is restricted to a certain range of deviations $d$ or low external fields, respectively. If a deviation of $5 \%$ from the linear slope is acceptable, the rigid vortex model remains valid in the example of Fig. 3 up to a vortex excursion of $0.7 r$, as can be seen from the graph.

Pinning effects can be studied by introducing a central hole into the circular disks, which acts as an artificial

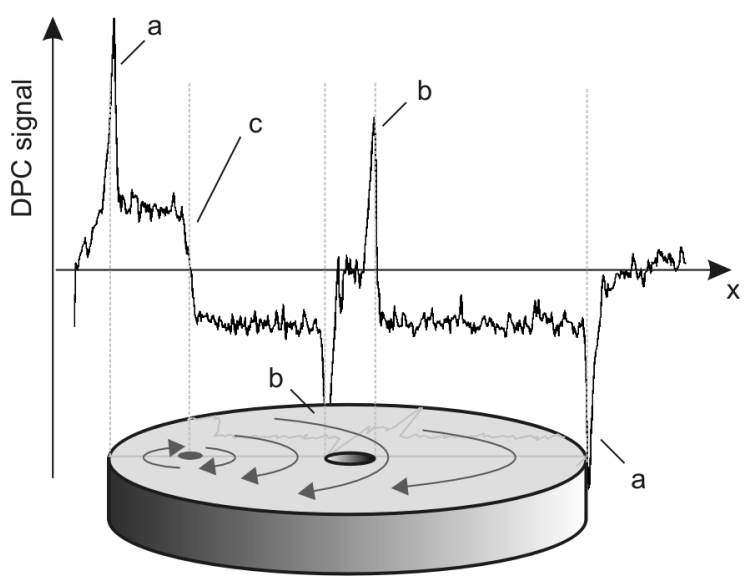

FIG. 2. The line scan method is shown, which allows the determination of the vortex core position. The outer (a) and inner (b) edges of the particle can be seen as sharp peaks in the signal, while the vortex appears as a change of the signal's sign. 


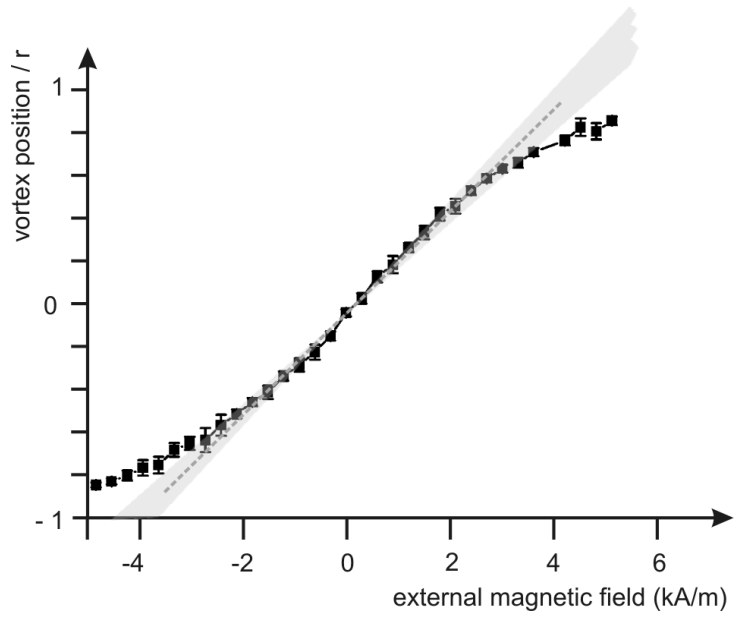

FIG. 3. The position of the vortex in a circular dot with $3 \mu \mathrm{m}$ diameter in an applied external field is depicted, the dashed line shows a linear fit in the zero field region, the gray area is the associated $5 \%$ confidence interval.

pinning site for magnetic structures. The holes are introduced as described above [8].

In Fig. 4 the vortex state was prepared in a ring structure with $3 \mu \mathrm{m}$ outer and $300 \mathrm{~nm}$ inner diameter by saturating the particle and subsequently decreasing the external field,
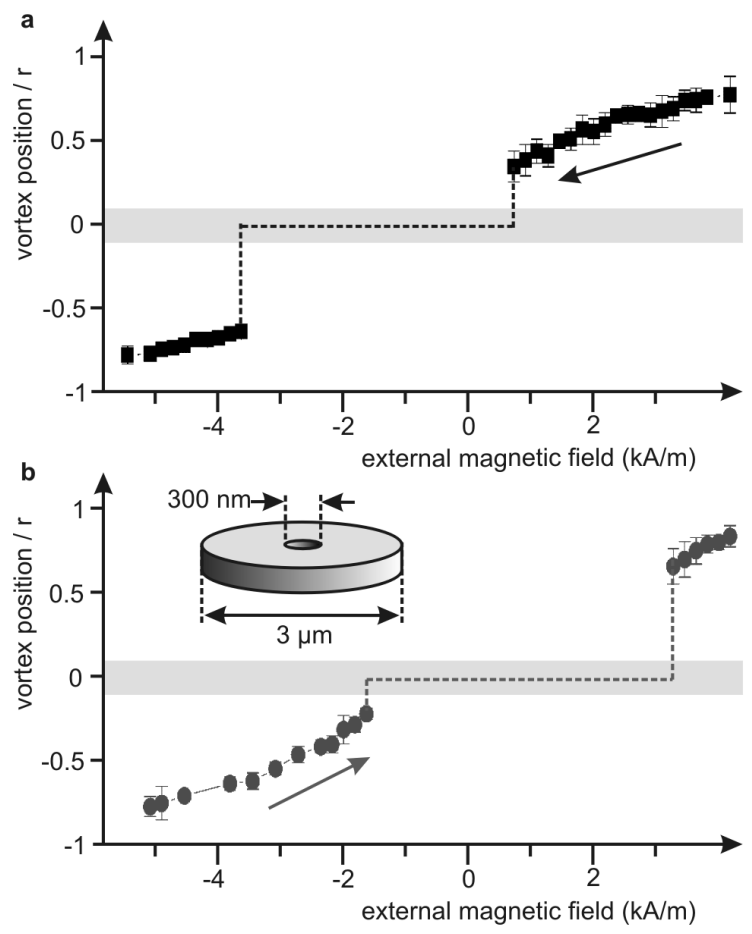

FIG. 4. A vortex configuration was prepared in a circular dot with $3 \mu \mathrm{m}$ diameter and a $300 \mathrm{~nm}$ central hole. The vortex was shifted by an external field towards the center from the right (a) or the left (b) side, respectively. In the central hole the vortex became pinned, before the hole boundary was actually reached, the vortex persisted pinned over a certain field range, until in the opposite field the vortex was released. until the vortex nucleates. Comparable to Fig. 3, the vortex was shifted by an external field from the outer region of the dot inwardly; the position is given as a relative position with respect to the diameter. Once the vortex comes closer to the hole edge than a certain distance, it jumps completely into the hole. This is energetically favored, because the center part of any vortex is an area of high exchange energy density. If the vortex core moves into the hole, this energy is not required any longer to build the vortex (outof-plane) core. That means, that an annihilation of the vortex core in the central hole can lead to a lower total energy of the particle. The vortex is stable over a certain field range, until it is depinned in a reversed external field.

Using our experimental setup it is possible to directly measure the depinning field strengths for various geometries. In the case presented here it turned out to be $-(3.6 \pm$ $0.2) \mathrm{kA} / \mathrm{m}$ and $(3.3 \pm 0.2) \mathrm{kA} / \mathrm{m}$ for opposite directions of the applied field.

In Fig. 5 intrinsic pinning in a circular dot of $1 \mu \mathrm{m}$ without artificial pinning sites has been observed. The vortex was shifted through the particle using an external field. The field-position plot shows several jumps, which can be attributed to the random pinning of the vortex on structural inhomogeneities in the structure. The enlargement shows clearly that the vortex position does not change over a certain magnetic field interval. Up to now it was not possible to assign the locations of pinning to certain distinctive structural features. This has to be the subject of future investigations.

It is, however, possible to give a qualitative explanation of this behavior. As the material investigated is polycrystalline, it consists of randomly assembled microcrystallites, each with a different easy magnetic axis due to its orientation. This causes the vortex to assume a position,

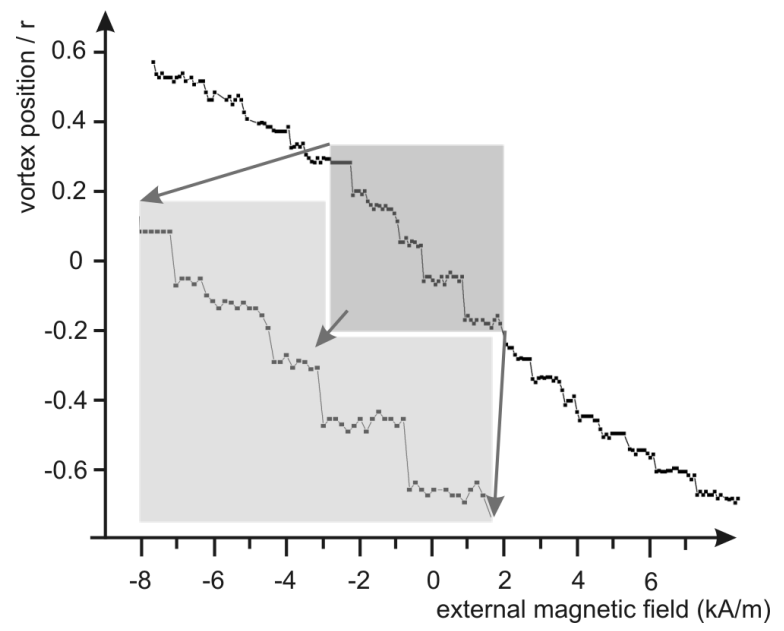

FIG. 5. The vortex is moved through a circular dot with a diameter of $1 \mu \mathrm{m}$. In this high-resolution measurement jumps in the vortex position are visible which can be attributed to microscopic pinning sites. The enlargement of the gray area shows this very clearly. 

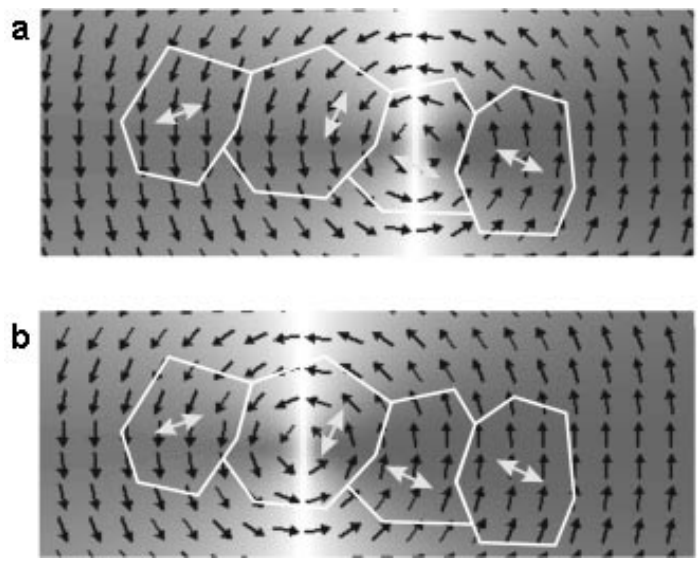

FIG. 6. Schematic representation of possible stable pinning sites for a vortex within a fictive crystallite arrangement. The double arrows give the local anisotropies within the crystallites, which are sought to be met as closely as possible by the internal structure of the vortex (black arrows indicate the local magnetization direction within the vortex, the gray scaled areas in the background represent predominantly down or up oriented regions). The vortex position is optimized to match the given local anisotropies with the least distortion and increase in the energies involved. The vortex position in (a) is energetically more favorable compared to that in (b), as the magnetization in one crystallite (second from left) corresponds to the respective anisotropy. Thus, pinning at the configuration (a) is expected.

where its magnetic structure is met best by the local anisotropies in order to minimize the total energy. This is achieved if a maximum volume fraction of the vortex is aligned with local easy axes of the surrounding crystallites. This is shown schematically in Fig. 6. Compared to Fig. 6(b), in Fig. 6(a) the magnetization pattern of the vortex fits well to the easy axis within one crystallite (second from left). If the vortex core is shifted to the left side [Fig. 6(b)], the magnetic anisotropy energy would rise, as the fraction of spins pointing in the easy axis direction decreases. Therefore, it is advantageous to maintain the configuration in Fig. 6(a) over a certain range of the external field and thus pinning occurs.

The mechanism described above will act as a pinning force, which has to be overcome before the vortex is freed to jump into the next local energy minimum position. From the shape of the curve in Fig. 5 (inset) it can then be concluded that many of the pinning sites are rather strong (deep local potential minima), because the vortex movement occurs mostly in jumps. Only after an increased magnetic field is able to move the vortex over the potential barrier separating two pinning sites, the vortex will move there and appear pinned again. When the pinning energy is lower, the increasing magnetic field causes a linear dis- placement of the vortex. In fact, this can also be observed in several areas.

In conclusion, it was shown for the first time that DPC allows the analysis of magnetic pinning behavior on a lateral length scale of at least $50 \mathrm{~nm}$. Direct experimental proof of the validity of the rigid vortex model has been given, together with an indication of its limits, giving experimental justification for the use of the rigid vortex model. For the first time, the vortex movement perturbed by artificial and intrinsic pinning in a circular dot was investigated with very high spatial resolution, which can be used to achieve detailed insight into pinning phenomena. Finally, a model for the local pinning and depinning behavior of vortices has been presented and discussed. The model is based on energy minimization in a locally varying environment of various crystalline anisotropies as found in polycrystalline specimens.

The authors acknowledge financial support by the German Research Society (DFG) (Forschergruppe 370 Ferromagnet-Halbleiter-Nanostrukturen).

*Present address: Deutsches Zentrum für Luft-und Raumfahrt, German Space Control Center, Oberpfaffenhofen, 82234 Weßling, Germany.

${ }^{\dagger}$ Corresponding author.

Electronic address: josef.zweck@physik.uni-regensburg. de

[1] A. Hubert and R. Schäfer, Magnetic Domains-The Analysis of Magnetic Microstructures (Springer-Verlag, Berlin 1998).

[2] S. Tehrani et al., J. Appl. Phys. 85, 5822 (1999).

[3] J. G. Zhu, Y. F. Zheng, and G. A. Prinz, J. Appl. Phys. 87, 6668 (2000).

[4] M. Kläui, C. A. F. Vaz, L. Lopez-Diaz, and J. A. C. Bland, J. Phys. Condens. Matter 15, R985 (2003).

[5] J. Rothman et al., Phys. Rev. Lett. 86, 1098 (2001).

[6] M. Kläui et al., Phys. Rev. B 68, 134426 (2003).

[7] J. Raabe et al., J. Appl. Phys. 88, 4437 (2000); T. Shinjo et al., Science 289, 930 (2000); M. Schneider, H. Hoffmann, and J. Zweck, Appl. Phys. Lett. 77, 2909 (2000).

[8] M. Rahm, J. Biberger, V. Umansky, and D. Weiss, J. Appl. Phys. 93, 7429 (2003).

[9] M. Rahm, J. Stahl, W. Wegscheider, and D. Weiss, Appl. Phys. Lett. 85, 1553 (2004).

[10] K. Yu. Guslienko et al., Phys. Rev. B 65, 024414 (2002).

[11] J. N. Chapman, J. Phys. D: Appl. Phys. 17, 623 (1984).

[12] R. Höllinger, http://www.opus-bayern.de/uni-regensburg/ volltexte/2004/387.

[13] A. Wachowiak, J. Wiebe, M. Bode, O. Pietzsch, M. Morgenstern, and R. Wiesendanger, Science 298, 577 (2002). 